\title{
Pandemic (H1N1) 2009 influenza vaccine uptake in pregnant women entering the 2010 influenza season in Western Australia
}

\author{
Scott W White, Rodney W Petersen and Julie A Quinlivan
}

$\mathrm{P}$ regnancy has been identified as a risk factor for severe pandemic (H1N1) 2009 influenza. ${ }^{1-3}$ Pregnant women were identified early in the pandemic as "vulnerable" and therefore candidates for heightened alertness to the disease. Once the H1N1-specific vaccine (CSL Limited, Melbourne, VIC) was available and judged by the Therapeutic Goods Administration as safe and effective, pregnant women were atop the list of recommended recipients in the first rollout of the vaccination program in September 2009. ${ }^{4}$ Indeed, pregnancy has been recommended as an indication for seasonal influenza vaccination since this category of vaccination was included in the Australian immunisation handbook in 2008. ${ }^{5}$ The Australian Chief Medical Officer wrote to general practitioners in November 2009 emphasising the increased risk to pregnant women of severe pandemic influenza and the suitability of the H1N1-specific vaccine for use in pregnancy. ${ }^{6}$

A widespread public education campaign was undertaken to alert those at increased risk of severe pandemic influenza to the recommendation for vaccination. In metropolitan Western Australia, individuals were directed to traditional immunisation providers such as their GPs or Aboriginal medical services. $^{7}$

Vaccination against pandemic influenza for pregnant women has been advocated by the Australian Technical Advisory Group on Immunisation, the World Health Organization, ${ }^{8}$ and the United States Centers for Disease Control and Prevention, ${ }^{9}$ on the basis that the theoretical risks associated with the vaccine are outweighed by the potential benefits to the pregnant woman and her fetus. After birth, immunity of the mother also reduces the risk of transmission to the infant who is too young to be vaccinated.

On informal questioning of patients at the public antenatal clinics at Joondalup Health Campus - a secondary hospital in WA we found that uptake of H1N1-specific vaccination among pregnant patients had been low. Therefore, we audited the uptake of H1N1-specific vaccination among pregnant patients, with the aim of identifying reasons for not receiving the vaccine.

\section{ABSTRACT}

Objective: To audit the uptake of pandemic (H1N1) 2009 influenza vaccine in pregnant women entering the 2010 influenza season in Western Australia, and to identify why some women did not receive the vaccine.

Design, setting and participants: Cross-sectional study of consecutive patients attending the Joondalup Health Campus public antenatal clinics in WA in January 2010.

Intervention: Audit of uptake of the H1N1-specific vaccine.

Main outcome measures: Rate of $\mathrm{H} 1 \mathrm{N1}$-specific vaccination, and reasons for not being the vaccinated.

Results: 479 of 541 women who attended the clinics (88.5\%) were included in the audit. Three women had been infected with pandemic influenza in the preceding influenza season, leaving 476 women who were eligible for vaccination in pregnancy. Of these 476 women, only 33 (6.9\%) had been vaccinated. Of the remaining 443 women who were eligible to receive the vaccine but had not been vaccinated, $63.9 \%$ had not been offered vaccination despite multiple visits to their general practitioners during pregnancy, $19.6 \%$ had been advised by their GPs against vaccination in pregnancy, and $61.6 \%$ stated that they would decline vaccination if offered because of safety concerns.

Conclusions: Uptake of $\mathrm{H} 1 \mathrm{~N} 1$-specific influenza vaccine in pregnant women was poor. Reasons for this relate both to vaccination not being offered to or actively sought by the women, as well as concerns - of both the women and their GPs - about vaccine safety in pregnancy. Uptake in this setting may improve if vaccination is offered through public antenatal clinics with concurrent safety education for obstetricians and vaccination providers.

MJA 2010; 193: 405-407

eMJA Rapid Online Publication 6 September 2010

\section{METHODS}

The study sample consisted of consecutive patients attending the Joondalup Health Campus public antenatal clinics over a 4week period in January 2010. Joondalup Health Campus services a metropolitanrural population mix (about two-thirds metropolitan).

During antenatal clinic consultations, patients were asked by their attending doctors whether they had received the "swine flu" (H1N1-specific) vaccine as prophylaxis against the pandemic influenza. If patients answered "no" to this question, they were asked why they had not been vaccinated. Patients could list multiple reasons. Immunisation outcomes for each patient - vaccination status (yes, no, previous infection) and, where applicable, response to the query as to why they had not received the vaccine - were entered into the medical record results summary page which was part of the patient's hospital medical record. Patients' immunisation outcomes were also recorded on a data sheet. Data sheets were collected by one of us (JAQ) at the end of each clinic session and were secured in a locked office within the Department of Obstetrics and Gynaecology.

After the first week, verbatim answers were evaluated using qualitative analytical methods ${ }^{10}$ and content analysis was undertaken independently by two of us (JA Q and RW P). This revealed three GP-related and two patient-related reasons for not receiving the vaccine. Both independent reviewers generated the same broad (GP and patient) categories and subcategories, meaning that there was high inter-rater reliability.

In the remaining 3 weeks of the audit, answers were coded to one of the subcategories determined by the qualitative analysis of the pilot data. In cases where patients were not able to articulate a reason for failure to accept vaccination, the responses were coded as "insufficient data to code".

The audit met the National Health and Medical Research Council (NHMRC) criteria 
for a negligible-risk project. The Joondalup Health Campus Human Research Ethics Committee exempts negligible-risk projects that meet the criteria outlined in section 5.1.22 of the NHMRC National statement on ethical conduct in human research from formal ethics approval processes. ${ }^{11}$

\section{RESULTS}

Of 564 patient bookings for the 4-week audit period, 23 were for patients who attended the antenatal clinics on a second occasion. This meant that 541 patients attended on at least one occasion, of whom 479 (88.5\%) were included in the audit. The remaining 62 patients were missed as the attending doctor forgot to ask and record responses to the audit questions. Similar numbers of patients were missed by each of the seven doctors who collected data during the audit.

Three patients included in the audit stated that they had been infected with "swine flu" in the preceding influenza season. Two of these cases had been confirmed by laboratory testing, and the other was presumptive - the patient developed symptoms after a family member had been microbiologically confirmed. This left 476 women who were eligible for vaccination in pregnancy. Of the 476 eligible women, only 33 women (6.9\%) had been vaccinated against pandemic influenza. The remaining 443 women were eligible to receive the vaccine but stated that they had not been vaccinated and, to the best of their knowledge, had not been infected in the preceding influenza season.

Qualitative and quantitative analysis identified five reasons why vaccination had not occurred: three related to the GP and two related to the patient (Box). The most common GP-related reason was that the GP had not raised or discussed the matter with the woman despite more than one visit during pregnancy (63.9\%). The most common patient-related reason was concern regarding safety of the vaccination in pregnancy $(61.6 \%)$. Of note, $19.6 \%$ of the women stated that their GPs had actively advised against vaccination.

\section{DISCUSSION}

This study showed poor uptake of H1N1specific vaccine $(6.9 \%)$ within a population of pregnant women in WA and identified several GP-related and patient-related reasons that influenced the poor uptake. This is despite previous findings reporting willingness to accept vaccination of $62.3 \%$ in Aus-

Reasons for not receiving the pandemic influenza vaccine, as given by pregnant women who were eligible to receive the vaccine $(n=443)^{*}$

Number (\%)

General practitioner-related reasons

Despite more than one visit to GP during pregnancy, GP has not suggested the vaccination to me

Visited GP once only during pregnancy (to confirm diagnosis and be referred to hospital for care) and the vaccination was not discussed during that visit

GP discussed the vaccination with me but advised against it during pregnancy

Insufficient data to code

\section{Patient-related reasons}

Personal safety concern, would decline the vaccination if offered

$273(61.6 \%)$

Did not think about the vaccination or raise the issue with my GP

$184(41.5 \%)$

Insufficient data to code

$22(5.0 \%)$

*Women could provide more than one reason for their answer.

tralian women and $64.6 \%$ in Australians aged 20-40 years. $^{12}$

Our findings highlight a suboptimal response to the education campaign on pandemic influenza: $41.5 \%$ of women had not thought about and sought vaccination, and $63.9 \%$ of women reported that their GPs had not raised the issue despite several consultations during pregnancy. Of more concern is that, even with specific communication from the Chief Medical Officer regarding the benefits and safety of vaccination in pregnancy, ${ }^{6} 19.6 \%$ of women reported active discouragement of vaccination by their GPs. Given the large number GPs who refer to the antenatal clinics at Joondalup Health Campus, it is likely this represents a number of different practices in the region. Also of note is that $61.6 \%$ of women expressed concern regarding the safety of the vaccine in pregnancy and stated that they would decline vaccination if offered. We wonder whether some of those women who expressed concern over safety may have been reassured by more effective education, including education of the GPs in the community who were advising against vaccination in pregnancy. GPs may feel more confident about offering H1N1-specific vaccination when it appears as a recommendation in literature from their local maternity hospital and is incorporated into the routine care plans for women, and especially when it is included in consensus statements for shared maternity care. Such strategies have been associated with high levels of support for and compliance with hepatitis B and anti-D vaccination.
This study has a number of limitations. First, demographic details were not collected, which makes generalisation of results difficult. However, the mean age at time of birth and proportion of primiparous women in the hospital population were similar to those reported for the wider Australian population - 30.4 years and 39\%, respectively, for the hospital population (Ms Wendy Candy, Deputy Director of Nursing [Midwifery], Joondalup Health Campus, personal communication, April 2010) v 29.9 years and $41.6 \%$, respectively, for the Australian population. ${ }^{13}$ The study design also meant that we were not able to determine how many women who were not offered vaccination by their GPs would have accepted vaccination had this occurred. In addition, some women who were eligible for inclusion in the audit were missed as their attending doctors forgot to ask and record responses to the audit questions, reflecting the busy nature of the clinic. Nonetheless, $88.5 \%$ of eligible patients were included, hence the results are representative of the antenatal clinic population.

Vaccination rates might be improved by shifting the provision of antepartum vaccination into antenatal clinics. Antenatal clinics are currently able to provide antenatal vaccination as prophylaxis against rhesus disease - this involves offering routine anti-D vaccination at 28 and 34 weeks' gestation to all rhesus-negative women. Maternity hospitals also provide routine vaccination against hepatitis B and rubella where indicated. Thus most hospital-based antenatal clinics should be able to initiate a suitable program 
to address pandemic influenza vaccination. It is possible that, in smaller clinics, patients could be vaccinated either in the clinic or referred to another vaccination provider if patient numbers are not sufficient to sustain an ongoing clinic-based program. This approach may also overcome difficulties experienced by obstetricians in private practice who may not be equipped to provide vaccination due to storage and cold-chain issues. Nevertheless, obstetricians are well suited to providing relevant education and could refer their patients to vaccination providers at other services.

Reasons for the poor uptake of H1N1specific influenza vaccine by pregnant patients at Joondalup Health Campus were related to GP and patient factors, including concerns regarding safety of vaccination in pregnancy. We believe that offering vaccination in antenatal clinics and improving safety education for obstetricians and vaccination providers could increase pandemic influenza vaccination rates.

\section{ACKNOWLEDGEMENTS}

We thank the staff in the antenatal clinics at Joondalup Health Campus for their assistance in data collection and the patients who contributed their information.

\section{COMPETING INTERESTS}

None identified.

\section{AUTHOR DETAILS}

Scott W White, MB BS, Obstetrics and Gynaecology Registrar ${ }^{1}$ Rodney W Petersen, MB BS, MBA, FRANZCOG, Director of Obstetrics and Gynaecology, $^{1}$ and Associate Professor ${ }^{2}$ Julie A Quinlivan, MB BS, PhD, FRANZCOG, Pro Vice Chancellor and Executive Dean of Medicine ${ }^{3}$

1 Joondalup Health Campus, Joondalup, WA.

2 Edith Cowan University, Joondalup, WA.

3 University of Notre Dame Australia,

Fremantle, WA.

Correspondence: juliequinlivan@nd.edu.au

\section{REFERENCES}

1 Jamieson DJ, Honein MA, Rasmussen SA, et al. H1N1 2009 influenza virus infection during pregnancy in the USA. Lancet 2009; 374: 451-458.

2 Hewagama S, Walker SP, Stuart RL, et al. 2009 H1N1 influenza $A$ and pregnancy outcomes in Victoria, Australia. Clin Infect Dis 2010; 50: 686690

3 Denholm JT, Gordon CL, Johnson PD, et al. Hospitalised adult patients with pandemic (H1N1) 2009 influenza in Melbourne, Australia. Med J Aust 2010; 192: 84-86.

4 Australian Government Department of Health and Ageing. Get vaccinated. http:// www.health.gov.au/internet/healthemergency/ publishing.nsf/Content/vaccine (accessed Feb 2010).
5 Australian Government Department of Health and Ageing. Australian immunisation handbook. 9th ed. Canberra: Australian Government, 2008.

6 Bishop J. 2 November 2009: letter to general practitioners on pregnant and postpartum women. http://www.health.gov.au/internet/ healthemergency/publishing.nsf/Content/prevadv-cmo (accessed Aug 2010).

7 Government of Western Australia Department of Health. Flu (influenza). http:// www.health.wa.gov.au/swine_flu/vaccination/ index.cfm (accessed Feb 2010).

8 World Health Organization. Safety of pandemic (H1N1) 2009 vaccines. 30 Oct 2009. http:// www.who.int/csr/disease/swineflu/frequently_ asked_questions/vaccine_preparedness/safety_ approval/en/ (accessed Feb 2010).

9 Centers for Disease Control and Prevention. 2009 H1N1 flu. http://www.cdc.gov/h1n1flu/ (accessed Feb 2010).

10 Neuendorf KA. The content analysis guidebook. London: Sage, 2002.

11 National Health and Medical Research Council. National statement on ethical conduct in human research. http://www.nhmrc.gov.au/_files_ nhmrc/file/publications/synopses/e72-jul09.pdf (accessed Jun 2010).

12 Eastwood K, Durrheim DN, Jones A, Butler M. Acceptance of pandemic (H1N1) 2009 influenza vaccination by the Australian public. Med J Aust 2010; 192: 33-36.

13 Laws P, Sullivan EA. Australia's mothers and babies 2007. Perinatal statistics series no. 23. (AlHW Cat. No. PER 48.) Sydney: National Perinatal Statistics Unit, Australian Institute of Health and Welfare, 2009. http://www.aihw.gov.au/publications/index.cfm/title/10972 (accessed Aug 2010).

(Received 23 Feb 2010, accepted 30 Jun 2010) 